An innovative FY3 post with 20\% QI time has been popular, with their on-call reducing locum spend.

Expansion of the non-medical workforce has begun with 5 permanent Physicians Assistants in paediatric specialties. There is expansion of The Advanced Clinical Practioner team into general paediatrics and surgery. These roles increase patient continuity and enable trainees to access educational opportunities.

Locum spend has significantly reduced with a $60 \%$ reduction in locum shifts.

This has been achieved with strong day-to-day leadership of the rotas, alongside a management team committed to improving staffing and working lives. Dedicated staff are essential for this strategy to be sustainable.

Challenges include significant ongoing time commitment, increased educational supervision and unpredictability of international recruitment.

We suggest that this model can be used by other UK Children's Hospitals and form an important part of a regional workforce strategy.

\section{G517(P) ABSTRACT WITHDRAWN}

\section{G518(P) ABSTRACT WITHDRAWN}

\section{British association of general paediatrics and YPHSIG (young people's health special interest group)}

\section{G519 INTEGRATED CHILD HEALTH AT SCALE: THE INREACH STORY - LESSONS LEARNT FROM THE DEVELOPMENT OF JOINT PAEDIATRIC CLINICS IN PRIMARY CARE}

${ }^{1,2} \mathrm{CP}$ Macaulay, ${ }^{1,2} \mathrm{C}$ Lemer, ${ }^{2} \mathrm{~S}$ Lamb, 1,21 Wolfe. 'Evelina London Children's Hospital, London, UK; ${ }^{2}$ Children and Young People's Health Partnership, London, UK

\subsection{6/archdischild-2020-rcpch.440}

Aims Over the last 3 years we have been developing and testing new models of integrated care for children and young people (CYP) in South London. As part of this we have developed a model of 'Inreach clinics' held in GP practices, jointly between GPs and Paediatricians. The overall aim is to improve care and outcomes for children. We report on our learning and successes of this journey.

Methods GP Practices were grouped into 'clusters' according to geography and population, each 'cluster' hosting one Inreach clinic per month. Initial challenges were commitment of GP time to host clinics, and shared patient notes and clinic management. Over time Paediatricians gained access to GP IT systems and notes were recorded locally. Lunch time 'lunch and learn' sessions for neighbouring practices after clinics were poorly attended so we developed the concept of a Patch Paediatrician (PP), giving GPs easy access for advice and referrals. We have honed referral pathways and introduced primary care prescribing by the PP. Clinics are currently jointly funded by the hospital and CCGs.
Results Currently 10 general paediatric consultants run monthly Inreach clinics in 16 patches, across 54 practices. We are conducting both service and research evaluations. Early results show reduction of ED attendances by $7 \%$ and NEL admissions by $6 \%$. Patient satisfaction data shows that the vast majority of patients feel that the care received was good or very good. GPs report enhanced knowledge, clinical practice and confidence in managing children's health.

Conclusions Over the last 2 years we have completed several PDSA cycles responding to challenges and feedback. We have demonstrated several successes of this model of care. Our current model aligns clinics and the role of 'patch paediatrician' to the newly created Primary Care Networks, for strong GP ownership. We are further aligning these clinics with our targeted population approach to biopsychosocial care for CYP with ongoing conditions. We are now evaluating, at scale, the best possible evidencebased model of care.

\section{G520 INNOVATIVE FUNDING STREAMS FOR CHILDREN WITH MEDICAL COMPLEXITY}

M Salama, MR Kelly. Children with Medical Complexities Team, Birmingham Children's Hospital, Birmingham, UK

\subsection{6/archdischild-2020-rcpch.441}

Aim The CMIC (Children with Medical Complexities) team is an innovative team which has been working within a tertiary children's hospital to support children with complex medical conditions who face a number of challenges. Our vision is to provide a pop up service tailored to the individual child and family. In doing so we support families navigating complex systems in a variety of ways. We have been fortunate to have a family support worker placed within our team for the last five years. She has been instrumental in providing support in many ways. One of the skills she has developed in the ability to look at third sector funded support to support both the child and family in getting home and staying home. In an increasingly cost driven system we looked at quantifying the monetary support she has been able to source in addition to the standard routes of disability living allowance and carer's allowance applications she helps families with as a routine.

Method We have looked back at the last 3 years of successful funding applications to third sector agencies. Results

Over the last 3 years our support worker has accessed funds from 6 additional charity funding streams. Some have been tailored to the individual child's condition whilst others provide more generic support. She has also managed to advocate for a family to have reimbursement of withheld carers allowance and personal independence payment. Over the three year period she has sourced a total of $£ 38500$. In addition to this she has been able to access experiences and pamper packs as well as smaller items for new homes and siblings.

Conclusion In an increasingly challenging financial environment, we demonstrate that with an innovative approach to provide direct support to families we can do more. We are using this knowledge and sharing it with stakeholders who support this group of children and families to further enable spread of this practice. 